\title{
The Danish polarimetric SAR for remote sensing applications
}

Christensen, Erik Lintz; Madsen, Søren Nørvang; Dall, Jørgen; Skou, Niels; Jørgensen, Jørn Hjelm; Woelders, Kim; Netterstrøm, A; Granholm, Johan; Dich, Mikael

\section{Published in:}

Proceedings of the International Geoscience and Remote Sensing Symposium

Link to article, DOI:

10.1109/IGARSS.1994.399439

Publication date:

1994

Document Version

Publisher's PDF, also known as Version of record

Link back to DTU Orbit

Citation $(A P A)$ :

Christensen, E. L., Madsen, S. N., Dall, J., Skou, N., Jørgensen, J. H., Woelders, K., Netterstrøm, A., Granholm, J., \& Dich, M. (1994). The Danish polarimetric SAR for remote sensing applications. In Proceedings of the International Geoscience and Remote Sensing Symposium: Surface and Atmospheric Remote Sensing: Technologies, Data Analysis and Interpretation (Vol. Volume 3, pp. 1361-1364). IEEE. https://doi.org/10.1109/IGARSS.1994.399439

\section{General rights}

Copyright and moral rights for the publications made accessible in the public portal are retained by the authors and/or other copyright owners and it is a condition of accessing publications that users recognise and abide by the legal requirements associated with these rights.

- Users may download and print one copy of any publication from the public portal for the purpose of private study or research.

- You may not further distribute the material or use it for any profit-making activity or commercial gain

- You may freely distribute the URL identifying the publication in the public portal 


\title{
The Danish Polarimetric SAR for Remote Sensing Applications
}

\author{
E. Lintz Christensen, S. Nørvang Madsen, J. Dall, N. Skou, \\ J. Hjelm Jørgensen, K. Woelders, A. Netterstrøm, J. Granholm, M.Dich \\ Electromagnetics Institute, Technical University of Denmark \\ B-348, Lundtoftevej 100, DK-2800 Lyngby, Denmark; Fax +45 45931634
} Phone +45 428814 44; E-mail Lintz@emi.dtu.dk

\section{ABSTRACT}

This paper presents the Danish polarimetric SAR system, EMISAR, and the approach taken in the system design to achieve a reliable high performance system. The design and implementation of the antenna system as well as the analog and digital hardware are discussed. The SAR utilises a dual polarised microstrip antenna with probe fed patches and the antenna exhibits a modified cosec-squared pattern with high polarisation discrimination. The two transmitted polarisations are time multiplexed at the transmitter and at the dual channel (V-and H-polar) receiver front end while azimuth pre-filtering is implemented by independent circuits for the four parallel channels ( $\mathrm{VV}, \mathrm{VH}, \mathrm{HV}$, and $\mathrm{HH}$ ). The system has been designed to permit both C- and L-band data to be recorded on the same high density digital tape. The paper presents the performance of the system including measured resolution, peak- and integrated sidclobes, polarimetric channel imbalance and cross-talk.

\section{INTRODUCTION}

Since 1989 Electromagnetics Institute (EMI) has flown a Cband, vertically polarised, Synthetic Aperture Radar (SAR) with a 2 by $2 \mathrm{~m}$ resolution and an $80 \mathrm{~km}$ range, [Madsen et al., 1991]. A full swath, full resolution real-time processor was completed in 1992, [Dall et al., 1992] and [Dall et al., 1993]. A fully polarimetric C-band system has since been implemented, and the first test flights took place in the fall of 1993. An additional L-band system with full polarimetric capability will be completed early 1995 .

The polarimetric SAR system is based on the single polarisation SAR which has been upgraded by adding and replacing the necessary components to achieve polarimetric capability. However, large parts of the digital electronics have also been upgraded to utilise new technology in order to gain a reduction in volume and power consumption. The polarimetric SAR thus requires less space and the same power as the previous single polarisation version.

The system is presently operated on a Gulfstream G-3 aircraft of the Royal Danish Air Force. The G-3 is a twin engine jet, with a $6000 \mathrm{~km}$ range. The SAR is nominally operated at an altitude of $41.000 \mathrm{ft}$. The antenna system and the radar INU are installed in a pod mounted below the fuselage, which facilitates rapid system installation and dismount. Typically the installation time is 2 hours.

The first test flights took place in September 1993 and in December 1993 the SAR was on a calibration mission to the DLR calibration site in Oberphaffenhofen, Germany. The performance of the system is presented in the paper including measured resolution, peak- and integrated sidelobes, uncalibrated and calibrated polarimetric channel imbalance and cross-talk. The data calibration is further discussed in [Dall et al. 1994] and [Skriver at al., 1994].

The SAR is presently being updated with interferometric capability for both cross track and along track interferometry.

One of the applications of the system is EMI's own radar research to be be carried out as part of the research plan for the
"Danish Center for Remote Sensing" which has recently been established at EMI by the Danish National Research Foundation. Furthermore, the upgrading to polarimetry and dual frequencies has been supported by Joint Research Centre (JRC) of the European Community and it is planned that EMI will operate the polarimetric SAR for JRC in connection with EARSEC (European Airborne Remote Sensing Capabilities), a remote sensing program managed and sponsored by JRC. Also, the sensor will be used in connection with EMAC (European Multi-sensor Airborne Campaigns) organised by ESA.

\section{SAR SYSTEM OVERVIEW}

A block diagram of the polarimetric SAR is shown in Fig. 1. Each of the shaded rectangles represents one physical unit. The Pod holding the 3-axis stabilised, dual polarised antenna (shown as two antennas to illustrate the principle) is mounted under the fusclage of the aircraft. The receiver and upconverter (RX-UPC) the high power transmitter (TX), the High Speed Digital Processor (HSDP), and the System Control Unit (SCU) are 19" boxes located in the aircraft cabin.

The function of the system can be explained as follows: before mapping a frequency modulated baseband pulse is computed and stored in the DSG buffer. At a $200 \mathrm{MHz}$ rate this pulse is D/A converted, I-Q modulated on an intermediate frequency carrier, up-converted to the SAR frequency, amplified, and finally transmitted by the antenna. After reflection the pulse is received, amplified, down-converted, demodulated and digitised. An optional range pre-filtering (RPF) can then be performed whereafter the peak data rate is reduced by the buffer in the DFE, thus facilitating the optional azimuth pre-filtering (APF). The pre-filtered data are recorded on a High Density Digital Tape (HDDT) as well as processed by the real-time processor (one channel only) for display on a monitor simultaneously with the data acquisition.

The H/V switch ensures that the pulses are alternately transmitted with horizontal and vertical polarisation. The transmitter branch of the polarimetric SAR is in principle identical to that of the single polarisation SAR. However, the receiver measures both $\mathrm{H}$ and $\mathrm{V}$ polarisation returns simultaneously for a total of four combinations of transmit and receive polarisations. The APF must thus be quadrupled because it combines consecutive pulses of like transmit and receive polarisation, [Skou, 1992].

A feature of the EMISAR is the internal calibration loops. In the normal mapping mode the signal from the upconverter is routed via the TWT to the polarisation switch to be radiated by the antenna. The received signals are routed to the dual receivers. Alternatively the receivers can be fed by a sample of the transmitter signal by changing the calibration switches. In a special calibration mode the signal from the upconverter is bypassing the TWT and routed directly via the polarisation switch towards the antenna where it is reflected, by means of shorting switches, back through the polarisation switch to the receivers. Again the receivers can alternatively be fed by a sample of the "transmitted" signal. By measuring the "received" signals in the 4 different modes the polarimetric calibration can be carried out for the complete system except the polarisation switch and the antenna, [Dall et al., 1994]. 


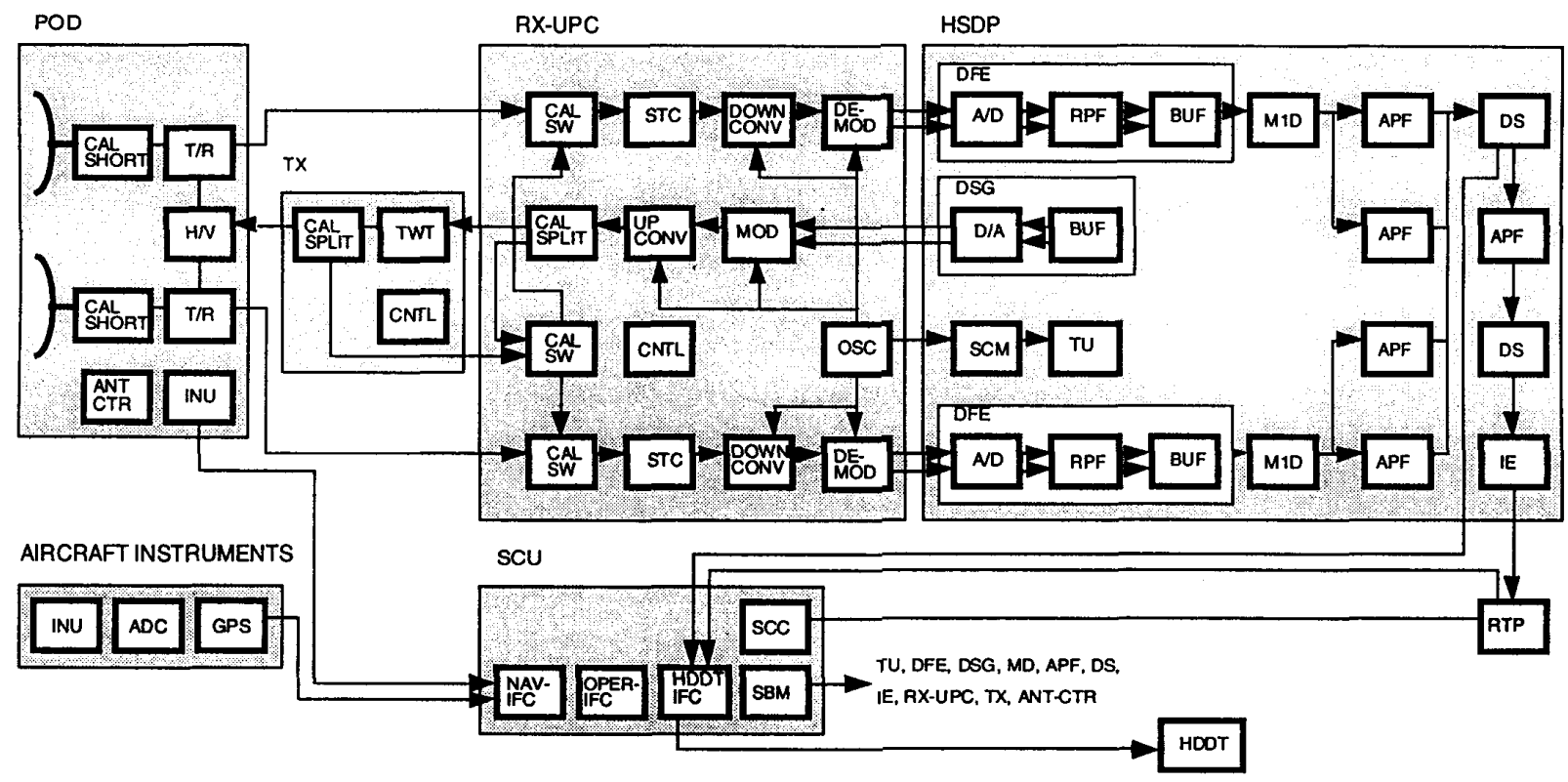

Fig. 1, EMISAR C-band polarimetric SAR overview diagram.

This calibration is carried out just before and after a mapping sequence, i.e. the calibration will be valid for the whole mapping if the SAR system is sufficiently stable for the few minutes of a normal data take. The analog subsystems denoted RX-UPC and TX are placed in thermally isolated and temperature controlled enciosures in order to achieve a high stability. A coarse temperature control of the POD has also been implemented. This is supposed to be adequate to achieve the necessary stability even for long data takes but whether this actually holds true also for the pod equipment cannot be stated with certainty until further tests have been completed and analysed.

\begin{tabular}{|l|c|}
\hline System Parameters: & C-Band $5.3 \mathrm{GHz}$ \\
\hline Transmitted power & $2 \mathrm{~kW}$ \\
\hline Receiver noise figure & $1.5 \mathrm{~dB}$ \\
\hline System loss & $4 \mathrm{~dB}$ \\
\hline Pulse length & $0.64-20 \mu \mathrm{s}$ \\
\hline Max. bandwidth & $100 \mathrm{MHz}$ \\
\hline Antenna gain & $25.7 \mathrm{dBi}$ \\
\hline Azimuth 3 dB beamwidth & $2.7^{\circ}$ \\
\hline Elevation pattern width & $30^{\circ}$ \\
\hline Polarisation & Fully polarimetric \\
\hline Antenna cross polarisation & $<-30 \mathrm{~dB}$ \\
\hline Azimuth ambiguity & $<-30 \mathrm{~dB}$ \\
\hline Resolution in range & 2,4 or $8 \mathrm{~m}$ \\
\hline Resolution in azimuth & 2,4 or $8 \mathrm{~m}$ \\
\hline Swath width & 12,24 or $48 \mathrm{~km}$ \\
\hline Range & max. $80 \mathrm{~km}$ \\
\hline PSLR & $<-30 \mathrm{~dB}$ \\
\hline ISLR & $<-25 \mathrm{~dB}$ \\
\hline Calibrated cross-talk terms & $<-35 \mathrm{~dB}$ \\
\hline Flight altitude & Full resolution \\
\hline Real-time processing, one channel & ically $41,000 \mathrm{ft}$ \\
\hline
\end{tabular}

Table 1, EMISAR C-band system parameters

\section{DIGITAL SUBSYSTEMS}

System Control Unit (SCU)

The polarimetric SAR is controlled by a central control unit consisting of a single board computer acting through a number of specialised interface modules all interconnected via a standard VME bus. The control system is highly automated. Under supervision of the operator it initiates self-test of the various system modules, sets up the complete system, and starts SAR calibration and data recording as programmed. The SCU also takes care of the antenna steering assuring optimum target area illumination and zero Doppler operation.

The NAV interface is used for collecting data from the aircraft instruments and for communication with the Inertial Navigation Unit (INU) situated in the pod, close to the antenna. The serial bus master (SBM) controls the operation of all the devices in the HSDP, RX-UPC, TX and POD. The HDDT interface controls the recording of radar data and ancillary data on tape.

\section{High Speed Digital Processor (HSDP)}

The main principles of the HSDP are based on the single polarisation SAR with the extensions required for full polarimetric operation. The design of the HSDP aimed at reducing the clock speeds through the parallelisation of the digital circuitry. The lower clock speeds allowed for the use of higher density integrated circuits (memories, EPLD's etc.) thereby resulting in smaller size while increasing functionality. Also, by using surface-mount component technology and densely packed multilayer printed circuit boards a substantial reduction in size was achicved.

The design allowed the removal of all high speed data connections between the subunits of the HSDP. This resulted in a simple and reliable low-speed interconnection backplane. The fully polarimetric HSDP only requires one 19 " box with a single power supply unit, whereas the single polarisation SAR HSDP required two 19" boxes and several power supply units.

The Digital Signal Generator (DSG) is a general purpose signal generator. The desired signals are downloaded in digital form and D/A converted during transmission. The DSG uses a phase twiddle strategy with $90^{\circ}$ phase increments on a pulse to pulse basis in order to compensate for imbalance and other deficiencies in the analog I/Q modulator and demodulator, [Netterstrøm and Christensen, 1994]. 
The range pre-filter (RPF) is a $100 \mathrm{MHz}, 71$ tap, digital FIR filter used to reduce the signal bandwidth and data rate, when resolution is traded for increased swath width. The filter performs a bandwidth reduction and decimation by a factor of 1,2 , or 4 . The azimuth pre-filter (APF) is a decimating integrate and dump filter which can decimate by a factor of 1 to 64 . The APF makes oversampling in azimuth, required to limit azimuth ambiguity, possible without overloading the HDDT.

The motion compensation module (M1D) carries out the first order motion compensation in order to assure that the azimuth signal spectrum is centred within the APF passband. In addition, the module removes the phase twiddle introduced by the DSG. A Doppler centroid estimator is a part of the module enabling online detection of static antenna pointing errors.

Real Time Processor (RTP)

The RTP is used to allow in-flight real time processing of a single polarimetric channel to full resolution. This enables a high degree of in-flight data verification and qualification. The RTP input may be switched to any of the four polarisations. The RTP consists of a local control computer and only 3 module types: a Programmable Digital Signal Processor, a Memory element, and an Interface element. Attached to the RTP is a Hardcopy unit for instant printout of quick-look images

\section{ANTENNA DESIGN}

The antenna has been designed to have a modified cosecsquared radiation pattern in elevation to allow for a large swath width with a fairly constant radar return. Furthermore the design has aimed at achieving low side lobes in both azimuth and elevation in order to keep the azimuth ambiguity low, [Christensen and Dich, 1993]. High polarisation discrimination was also a priority design parameter.

The antenna is built using microstrip technology, [Woelders and Granholm, 1992] and [Granholm et al., 1994]. The antenna is manufactured by 4 identical panels. It has a total size of $1.35 \mathrm{x}$ $0.35 \mathrm{~m}$ and is designed for the frequency band $5.3 \mathrm{GHz} \pm 50$ $\mathrm{MHz}$. Each panel has 7 square microstrip patches in the elevation direction and 8 patches in the azimuth direction. The 8 columns of patches on each panel have equal excitation. The pattern in elevation has been shaped by a suitable excitation of the 7 rows of patches. The pattern in azimuth has been shaped to reduce the sidelobes by the excitation of the 4 individual panels.

Each panel is constructed like a sandwich with a silver plated ground plane in the middle. The radiating part is a microstrip circuit on a $2.0 \mathrm{~mm}$ low permittivity Rohacell $31 \mathrm{HF}$ substrate placed on one side of the ground plane while the feed network is a microstrip circuit on $0.787 \mathrm{~mm} \mathrm{RT/duroid} 5870$ on the other side. The radiating patches are connected to the V-port and H-port feed networks by probes passing through holes in the groundplane.

By this construction the complete feed network is on the backside of the antenna and this prevents radiation from the feed network which would otherwise contribute to the cross polarisation. Radiation from the probes and coupling between the probes cannot be completely avoided by this construction. The V-port network is a straightforward resonant design with each patch being fed with signals in phase. The $\mathrm{H}$-port network divides the 8 patches in a row in two groups. Every second patch is fed in anti phase with the others but the probe is connected on opposite locations of the patches to give the same patch excitation.

The result of this is almost complete elimination of coupling between the probes. The direct radiation from the probes has a null in the broadside direction and since the azimuth main lobe is narrow there is virtually no cross polar radiation from the V-port probes in the main lobe. The elevation lobe is wide but the phase alternation of the $\mathrm{H}$-port probes causes a significant reduction of the cross polar radiation from these in the elevation main lobe. The penalty is two side lobes in azimuth at $+/-45$ degrees, $20 \mathrm{~dB}$ below the main lobe. These sidelobes contribute to the azimuth ambiguity.

The azimuth and elevation patterns are applied in the absolute calibration of the SAR system. The measured antenna directivity in azimuth and elevation are given in figures 2 and 3 showing both the H-port co-polar and the cross-polar patterns. The V-port patterns are almost identical to these [Granholm et al., 1994]. The antenna maximum gain is $25.7 \mathrm{~dB}$ and the polarisation discrimination of the antenna is better than $-30 \mathrm{~dB}$ in the main lobe.

The fact that the antenna illuminates targets with Doppler shifts outside the processed part of the Doppler spectrum causes azimuth ambiguity. The integrated azimuth ambiguity to signal ratio has been calculated from the antenna pattern to be $-31.1 \mathrm{~dB}$ for horizontal polarisation and $-32.7 \mathrm{~dB}$ for vertical polarisation where the difference is caused by the $\pm 45^{\circ}$ side lobes. Azimuth ambiguity responses from individual point targets (ghost echoes) will be suppressed by at least the two-way attenuation of the side lobe directivity, i.e. more than $37 \mathrm{~dB}$.

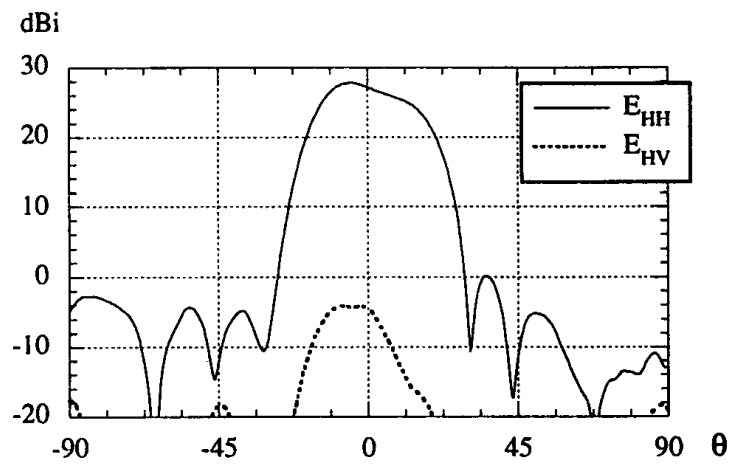

Fig. 2, Measured antenna $\mathrm{H}$-port co- and cross polar directivity versus elevation angle; sampling $0.5^{\circ}$.

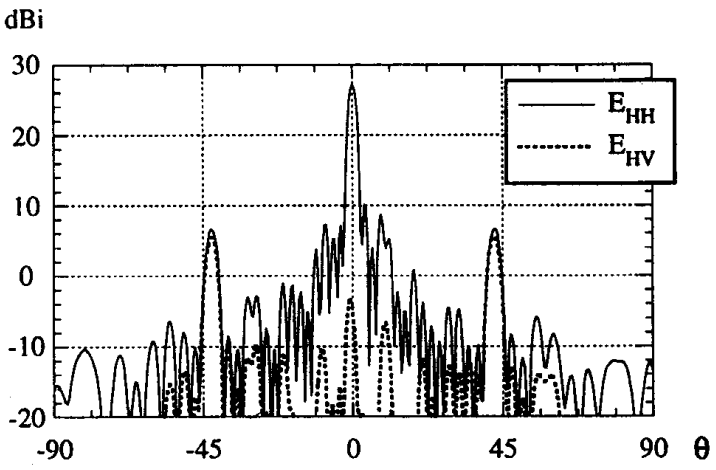

Fig. 3, Measured antenna $\mathrm{H}$-port co- and cross polar directivity versus azimuth angle; sampling $0.5^{\circ}$.

\section{PERFORMANCE EVALUATION}

The evaluation of the SAR has been performed during various test flights including an EMAC calibration experiment over Oberpfaffenhofen, Germany. Some of the procedures of this calibration is described in [Dall et al., 1994] and [Skriver et al., 1994]. The measured azimuth and range impulse responses are summarised in Table 2.

\begin{tabular}{|c|c|c|c|c|}
\hline & $\begin{array}{c}\text { azimuth } \\
\mathrm{HH}\end{array}$ & $\begin{array}{c}\text { azimuth } \\
\text { VV }\end{array}$ & $\begin{array}{c}\text { range } \\
\mathrm{HH}\end{array}$ & $\begin{array}{c}\text { range } \\
\mathrm{VV}\end{array}$ \\
\hline $3 \mathrm{~dB}$ resolution $[\mathrm{m}]$ & 1.97 & 1.98 & 2.09 & 2.08 \\
\hline PSLR & -35.2 & -33.8 & -34.2 & -30.8 \\
\hline ISLR & -29.1 & -28.6 & -30.2 & -30.2 \\
\hline
\end{tabular}

Table 2. Impulse response parameters measured for $2 \mathrm{~m}$ trihedral. 
In order to estimate the channel imbalance and the radiometric calibration parameters the measured scattering matrix is modelled as:

$\mathbf{M}=k\left[\begin{array}{cc}1 & 0 \\ 0 & f_{r x}\end{array}\right]\left[\begin{array}{cc}1 & \delta_{r 1} \\ \delta_{r 2} & 1\end{array}\right]\left[\begin{array}{ll}S_{h h} & S_{h v} \\ S_{v h} & S_{v v}\end{array}\right]\left[\begin{array}{cc}1 & \delta_{t 2} \\ \delta_{t 1} & 1\end{array}\right]\left[\begin{array}{cc}1 & 0 \\ 0 & f_{l x}\end{array}\right]$

where the SAR deficiencies are represented by a channel imbalance matrix and a cross-talk matrix for both the transmitter and the receiver branch.

The channel imbalance parameters were estimated using 9 trihedrals and 4 dihedrals deployed at the Oberpfaffenhofen site. The average and standard deviations of the results are:

$\left|f_{t x}\right|=-0.13 \mathrm{~dB} \pm 0.28 \mathrm{~dB}, \quad \angle f_{l x}=11.0^{\circ} \pm 2.9^{\circ}$

$\left|f_{r x}\right|=0.94 \mathrm{~dB} \pm 0.27 \mathrm{~dB}, \quad \angle f_{r x}=133.1^{\circ} \pm 3.5^{\circ}$

The absolute radiometric calibration was assessed for each of the trihedrals by comparing the measured signals with the radar cross section expected when correcting for the mismatch of the SAR and reflector depression angles. The average and standard deviation for the calibration constant was measured to:

$|k|=-1.95 \mathrm{~dB} \pm 0.34 \mathrm{~dB}$

Cross-talk calibration was carried out using distributed targets in the scene for which the true co- and cross-polarised returns are assumed to be uncorrelated, [Skriver et al., 1994]. The algorithm estimates the four cross-talk terms after the channel imbalance correction:

$u=\delta_{r 2}, \quad v=\delta_{l !}, \quad w=\delta_{r 1}, \quad z=\delta_{l 2}$

The cross talk terms, being below $-30 \mathrm{~dB}$ before calibration, were improved $8 \mathrm{~dB}$ by the calibration to be below $-38 \mathrm{~dB}$ worst case and typically around $-42 \mathrm{~dB}$ for the scene being analysed.

\section{ONGOING ENHANCEMENTS}

The work on an L-band SAR is in progress and is planned to be ready for the first test flight by the end of 1994 . The $\mathrm{L}-$ and $\mathrm{C}$ band digital subsystems will be identical and the two systems will share the same HDDT when the two frequencies are used simultaneously. The HDDT data rate of $240 \mathrm{Mbit} / \mathrm{s}$ is sufficient for 8 polarimetric channels with 8192 complex samples in range for each $1.5 \mathrm{~m}$ in azimuth. The analog subsystem will be made according to the same principles as the C-band but the lower frequency will influence the actual design. The transmitter power will be higher $(6 \mathrm{~kW})$ than the C-band and the antenna gain will be lower, since the two antennas will have approximately the same size. The design goal is to get the same bandwidth for L-band as for C-band.

An interferometric C-band system is under development using the existing system components wherever possible. The present $C$ band SAR will be used as the transmitter while two receive-only subsystems will be added to collect the cross track interferometric signals.

The baseline which can be obtained by antennas on an aircraft is obviously limited and this in turn limits the interferometric resolution. The higher resolution which can be achieved by repeat track interferometry will be pursued. The present aircraft P-code GPS is used for manual navigation only. The SCU has access to both GPS and INU and preparations are in progress to get the SCU connected to the auto-pilot for improved flight accuracy.

\section{CONCLUSION}

The preliminary evaluation of our polarimetric SAR indicates that the system meets or exceeds the design goals for all critical parameters, including the resolution, PSLR, ISLR and cross polarisation.

The internal calibration was expected to remove almost all of the uncertainties in the system parameters. However, these preliminary results indicates there are some deviations between the internal and the external calibration. If the system relied on internal calibration, the external calibration indicated an absolute calibration error of $-1.9 \mathrm{~dB}$ and an imbalance of $0.8-0.9 \mathrm{~dB}$, which is not considered satisfactory. However, this is of little importance for the user as long as the stability of the calibration is adequate but that must be verified through continued monitoring over several campaigns.

A notable feature of the EMISAR is the use of digital code generation and the extended use of digital signal processing; benefits including the flexibility in resolution and swath width, the possibility for compensating for distortion from the analog system, and the option of applying different transmitted signal waveforms. These features make the EMISAR well suited for radar research.

\section{ACKNOWLEDGEMENT}

A SAR system is not produced overnight, and many persons have been involved in the design and construction of the polarimetric SAR presented in the present paper. The authors want in particular to acknowledge S. Savstrup Kristensen and J. Grinder Petersen (off-line processing), H. Skriver (polarimetric calibration), N. Andersen and N. Vardi (hardware implementation), and B. Brandstrup and K. Christensen (technical support).

Development of the EMISAR system has been co-sponsored by the Thomas B. Thriges Foundation, the Danish Technical Research Council (STVF), the Royal Danish Air Force (RDAF), the Technical University of Denmark and the Joint Research Centre (JRC). Data acquisitions have been sponsored by the RDAF, STVF, JRC, and the European Space Agency, ESA.

\section{REFERENCES}

Madsen, S.N., Christensen, E.L., Skou, N., and Dall, J.

The Danish SAR System; Design and Initial Tests

IEEE Transactions on Geoscience and Remote Sensing, Vol 29 No 3, May 1991, pp 417-426.

Dall, J., Jørgensen, J.H., Christensen, E.L., and Madsen, S.N. Real-Time Processor for the Danish Airborne SAR

IEE Proceedings Part F, Special part on Remote Sensing, pp 115-121, April 1992

Dall, J., Jørgensen, J.H., Netterstrøm, A., Vardi, N., Christensen, E.L., and Madsen, S.N

The Danish Real-Time SAR Processor: First Results IGARSS '93, 1993 International Geoscience and Remote Sensing Symposium, Tokyo, August 18-21, 1993, Vol. 3, pp 14011403.

Dall, J., Skou, N., and Christensen, E.L. Pulse based Internal Calibration of Polarimetric SAR IGARSS '94, 1994 International Geoscience and Remote Sensing Symposium, California, USA, 8-12 August 1994.

Skriver, H., Dall, J., and Madsen, S.N.

External Polarimetric Calibration of the Danish Polarimetric Cband SAR

IGARSS '94, 1994 International Geoscience and Remote Sensing Symposium, California, USA, 8-12 August 1994.

Skou, N.

The Danish Polarimetric SAR System

Second International Workshop on Radar Polarimetry, Nantes, France, 8-10. September 1992, pp 525-533.

Netterstrøm, A., and Christensen, E.L. Correction for Quadrature Emors IGARSS '94, 1994 International Geoscience and Remote Sensing Symposium, California, USA, 8-12 August 1994.

Christensen, E.L., and Dich, $M$. SAR Antenna Design for Ambiguity- and Multipath SuppresSion

IGARSS '93, 1993 International Geoscience and Remote Sensing Symposium, Tokyo, August 18-21, 1993, Vol. 2, pp 784787 .

Woelders, K., and Granholm, J.

Design and Performance of a Dual-Linearly Polarized C-band Microstrip Array Antenna

Second International Workshop on Radar Polarimetry, Nantes, France, 8-10. September 1992, pp 369-378.

Granholm, J., Woelders, K., Dich, M., and Christensen, E.L. Microstrip Antenna for Polarimetric C-band SAR IEEE AP-S International Symposium and URSI Radio Science Meeting, Seattle, Washington, June 19-24, 1994 